\title{
Substratos a base de esterco de animais para produção de mudas de mamoeiro
}

\author{
Substrates based on animal manure for production of papaya seedlings
}

Karinne Silva do Nascimento 1

Engenheira Agrônoma, Instituto Federal de Educação, Ciências e Tecnologia do Piauí, Campus Oeiras,

E-mail: karinneagronoma@hotmail.com

João Afonso Nunes da Cunha Junior 2

Técnico em Agricultura, Instituto Federal de Educação, Ciências e Tecnologia do Piauí, Campus Oeiras E-mail: joaoafonsojunior3@gmail.com

Júlio Ferreira de Souza Filho 3

Engenheiro Agrônomo, Instituto Federal de Educação, Ciências e Tecnologia do Piauí, Campus Oeiras E-mail: julio.filho@ifpi.edu.br

Marcondes Araújo da Silva 4

Prof. de Agricultura do Instituto Federal de Educação, Ciências e Tecnologia do Piauí, Campus Oeiras

E-mail: marcondes.araujo@ifpi.edu.br

\begin{abstract}
Resumo: Para obtenção de pomares vigorosos e sadios é necessário a produção de mudas com qualidade, e menor custo de formação, observando o mais adequado substrato utilizado, pois o mesmo deve ter características que permitam a boa formação e desenvolvimento da muda. Objetivou-se nesse trabalho avaliar o desenvolvimento de mudas de mamoeiro "Formosa" e "Hawaí" submetidas a diferentes dosagens de esterco bovino, ovino e caprino. O Experimento foi conduzido em casa de vegetação situada na Unidade de Aulas Práticas e Pesquisa (UAPP) do Instituto Federal de Educação, Ciência e Tecnológica do Piauí (Campus Oeiras). As sementes foram semeadas em copos de polietileno com capacidade de 0,5 L, colocando-se duas sementes por recipiente. As mudas foram desbastadas, deixando-se apenas a mais vigorosa por recipiente. A avaliação foi 70 dias após a semeadura, considerando-se as variáveis tais como: porcentagem de germinação, a altura linear das mudas $(\mathrm{cm})$, diâmetro do colo $(\mathrm{mm})$, número de folhas; matéria fresca da parte aérea $(\mathrm{g})$, matéria fresca do sistema radicular $(\mathrm{g})$, matéria seca da parte aérea (g), matéria seca do sistema radicular. Foram obtidos resultados satisfatórios nos tratamentos T5 e T8 composto de esterco ovino (1:1) e (somente esterco ovino) e T12 (somente esterco caprino).
\end{abstract}

Palavras-chave: Papaya. Substrato. Esterco de Curral.

Abstract: In order to obtain vigorous and healthy orchards, it is necessary to produce seedlings with quality and lower cost of formation, observing the most appropriate type of container and substrate used, since it must have characteristics that allow the proper formation and development of the seedlings. The objective of this work was to evaluate the development of "Formosa" and "Hawaii" papaya seedlings submitted to different dosages of bovine, ovine and caprine manure. The experiment was conducted in a greenhouse located in the Unit of Practical Classes and Research (UAPP) of the Federal Institute of Education, Science and Technology of Piauí (Campus Oeiras). The seeds were seeded in 0,5 L polyethylene cups, with two seeds per container. The seedlings were thinned, leaving only the most vigorous per container. The 
evaluation was 70 days after sowing, considering characteristics such as: percentage of germination, linear height of the seedlings $(\mathrm{cm})$, diameter of the collar $(\mathrm{mm})$, number of leaves; Fresh matter of the aerial part $(\mathrm{g})$, fresh matter of the root system $(\mathrm{g})$, dry matter of the aerial part (g), dry matter of the root system. Satisfactory results were obtained in treatments T5 and T8 composed of sheep manure (1:1) and (only sheep manure) and T12 (goat manure only).

Keywords: Papaya. Substrate. Curral manure.

\section{Introdução}

O mamoeiro (Carica papaya L.) é uma planta originária da América Tropical, que pertence à família Caricaceae, essa cultura tem significativa participação na produção nacional de frutas, com maior rendimento $\left(48 \mathrm{t} \mathrm{ha}^{-1}\right)$ e a sexta mais produzida $(1,4$ mil t) (ANUÁRIO, 2015), pois é uma das mais consumidas no Brasil e apresenta grande relevância econômica e social, principalmente na geração de emprego e renda (GALO et al., 2014).

É uma cultura amplamente difundida e apreciada nas mais diversas partes do mundo, sendo cultivada em diversos países localizados nas regiões tropical e subtropical, tendo como principal produto comercializado o fruto, que devido às suas características nutricionais tem ganhado cada vez mais notoriedade no mundo (LIMA, 2014; PINTO, 2013).

De acordo com Vallone (2009), para a exploração comercial de uma cultura é indispensável que todas as etapas sejam bem planejadas e executadas, desde a produção das mudas até a formação do pomar, já que qualquer erro pode comprometer o desenvolvimento das plantas e a produtividade, consequentemente, o sucesso da implantação do pomar.

O emprego de mudas de qualidade se torna um fator decisivo na implantação da cultura, devendo-se atentar para o fato de que mudas de qualidade são aquelas que sobrevivem e se desenvolvem bem após o transplantio, isentas de patógenos e pragas, sendo produzidas a baixo custo, em recipiente com substrato que permita a boa formação e desenvolvimento da muda (JOHNSON et al., 1991).

O substrato é um insumo importante dentro do sistema de produção de mudas que podem ser formados por diferentes matérias-primas (ABREU et al., 2002), sendo que para a formação de mudas de mamoeiro, várias misturas são sugeridas, como a combinação entre solo, esterco, areia, entre outros, nas mais diversas proporções, visto que o esterco bovino é o mais usado e que tem demonstrado bons resultados na produção de mudas (TRINDADE, 1999), um exemplo disso são os estudos desenvolvidos por Lima et al., (2011) ao testar diferentes dosagens de esterco bovino para produção de mudas do mamoeiro.

A composição do substrato deve ser feita de acordo com a disponibilidade de materiais na propriedade e as características apresentadas pelo mesmo, observando as recomendações 
(SILVA et al., 2001), onde os substratos devem apresentar, entre outras importantes características, ausência de patógenos, riqueza em nutrientes essenciais, $\mathrm{pH}$ adequado, boa textura e estrutura que permita a retirado do torrão facilmente e intacto para não deformar o sistema radicular (GOMES; COUTO, 1986).

Diante do exposto acima, o presente trabalho teve como objetivo avaliar o desenvolvimento de mudas de mamoeiro das variedades "Formosa" e "Hawaí" roduzidas em substrato composto por diferentes dosagens de esterco bovino, ovino e caprino.

\section{Metodologia}

Local do experimento

O experimento foi conduzido no período entre novembro de 2016 a janeiro de 2017, em casa de vegetação da Unidade de Aulas Práticas e Pesquisa (UAPP) do Instituto Federal de Educação, Ciência e Tecnológica do Piauí - Campus Oeiras, no município de Oeiras, Piauí. Oeiras localiza-se a uma latitude $07^{\circ} 01^{\prime} 30$ " sul e a uma longitude $42^{\circ} 07^{\prime} 51^{\prime \prime}$ oeste e apresenta tipo climático predominante semiárido piauiense com clima subúmido seco, segundo Thornthwaite (1948) apud Andrade Junior (2004). Com altitude de 167m, temperatura média durante a execução do experimento variou de 25 a $33^{\circ} \mathrm{C}$, com precipitação média de $51,92 \mathrm{~mm}$ e umidade relativa em torno de $75 \%$.

Condução do experimento

Foi utilizado o delineamento experimental inteiramente ao acaso com arranjo fatorial 2x13 (13 tratamentos e 2 variedades de mamão) com cinco repetições. Os tratamentos são as seguintes proporções de solo e esterco de animal: $\mathrm{T}_{1}$ : Solo + esterco bovino (1:1); $\mathrm{T}_{2}$ : Solo + esterco bovino (2:1); $\mathrm{T}_{3}$ : Solo + esterco bovino (3:1); $\mathrm{T}_{4}$ : Esterco bovino; $\mathrm{T}_{5}$ : Solo + esterco ovino (1:1); $\mathrm{T}_{6}$ : Solo + esterco ovino (2:1); $\mathrm{T}_{7}$ : Solo + esterco ovino (3:1); $\mathrm{T}_{8}$ : Esterco ovino; $\mathrm{T}_{9}$ : Solo + esterco caprino (1:1); $\mathrm{T}_{10}:$ Solo + esterco caprino (2:1); $\mathrm{T}_{11}:$ Solo + esterco caprino (3:1); $\mathrm{T}_{12}$ : Esterco caprino; $\mathrm{T}_{13}$ : Solo. Para cada tratamento foi utilizado duas variedades de mamão (Formosa e Hawaí). Os estercos foram curtidos por 30 dias antes da formação dos substratos.

As sementes utilizadas foram produzidas pela empresa Feltrim ${ }^{\circledR}$ dos grupos: "Solo" (Hawaí) e Formosa, e foram semeadas em copos de polietileno com capacidade de 0,5 L, 
colocando-se duas sementes por copo. O desbaste das mudas foi realizado aos 30 dias após a emergência das plantas, deixando-se apenas a mais vigorosa por recipiente. Foram realizadas cinco adubações via foliar com Vitan ${ }^{\circledR}$ (composição: 6,5\% de $\mathrm{N} ; 8 \%$ de $\mathrm{P}_{2} \mathrm{O}_{5} ; 8 \%$ de $\mathrm{K}_{2} \mathrm{O}$; 0,5\% de $\mathrm{Mg} ; 1 \%$ de $\mathrm{Ca} ; 1 \%$ de $\mathrm{Zn} ; 0,6 \%$ de $\mathrm{B}$; e $0,5 \%$ de $\mathrm{Mn}$ ), para o suprimento nutricional das mudas de mamoeiro. Aplicações periódicas com fungicidas e inseticidas foram realizadas visando a sanidade das mudas de acordo com as recomendações dos produtos.

Avaliação do experimento

Foram avaliados: aos 15 dias após a semeadura (DAS) a porcentagem de germinação e aos 70 DAS, os seguintes parâmetros: altura linear das mudas $(\mathrm{cm})$, diâmetro do colo $(\mathrm{mm})$, número de folhas; matéria fresca da parte aérea $(\mathrm{g})$, matéria fresca do sistema radicular $(\mathrm{g})$, matéria seca da parte aérea $(\mathrm{g})$, matéria seca do sistema radicular $(\mathrm{g})$. A determinação da altura da muda foi realizada com uma régua graduada em centímetro, medindo a distância entre o colo e o ápice da muda. O diâmetro do calcule foi feito através de paquímetro digital, a matéria seca da raiz e da parte aérea foi obtida após secagem em estufa com circulação forçada de ar a 65 ${ }^{\circ} \mathrm{C}$, até atingirem peso constante, procedendo à pesagem em balança analítica.

Os dados obtidos foram submetidos à análise de variância, e as médias, ao teste de Scott Knott, a 5\% de probabilidade, utilizando o programa Sisvar® versão 5.4 para a comparação das médias (FERREIRA, 2010).

\section{Resultados e Discussão}

A germinação das sementes iniciou-se aos nove DAS, estendendo-se por aproximadamente 15 dias. A Figura 1 mostra o percentual de germinação. 
Figura 1. Germinação das sementes de mamão Hawai e Formosa submetidas a diferentes doses de estercos bovino, ovino e caprino.

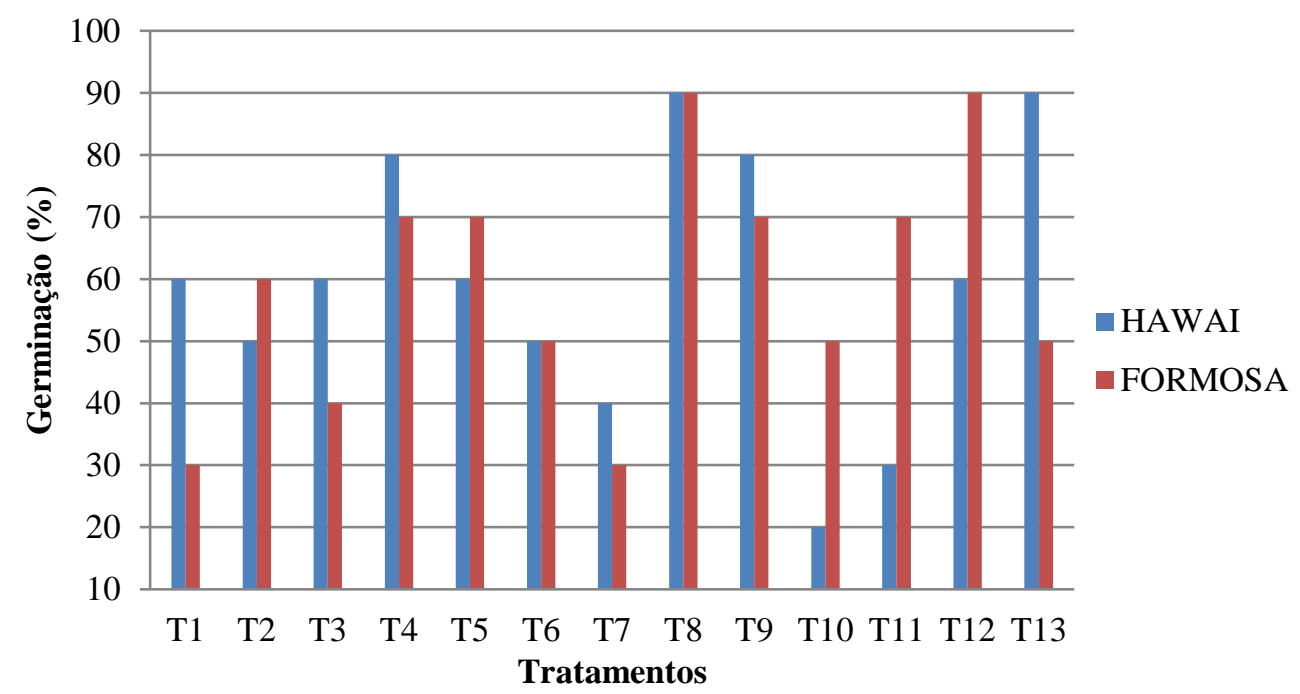

A variedade Hawaí, apresentou a menor taxa de germinação no T10, já a variedade formosa teve baixa germinação no $\mathrm{T} 1$ e $\mathrm{T} 7$, os tratamentos $\mathrm{T} 8$ e $\mathrm{T} 13$ proporcionaram as maiores taxas germinativas da variedade Hawaí, já a Formosa obteve melhor resultado nos tratamentos T8 e T12. Nota-se que T8 apresentou a melhor porcentagem de germinação para ambas as variedades.

Oliveira Filho et al. (2013), avaliando o efeito de diferentes fontes e doses de fertilizantes orgânicos na germinação e crescimento de mudas de mamoeiro "Formosa" em tubetes, observaram bons resultados quando se utilizou o esterco de ovino nas doses entre $40 \%$ e $45 \%$ do substrato. Oliveira et al. (2015) argumenta em seu estudo que estes bons resultados alcançados quando usado doses de esterco ovino são explicados pela influência direta da matéria orgânica na retenção de umidade nestes substratos, favorecendo, assim, maior embebição da água pelas sementes e, por conseguinte, maiores taxas de germinação.

As mudas de mamoeiro Hawaí apresentaram melhor altura no T8, diferindo estatisticamente dos demais, já as mudas de mamoeiro Formosa se desenvolveram melhor no T5, não diferindo do T8; os tratamentos T1, T2 e T3, apresentaram as menores alturas das mudas das variedades estudadas (Tabela 1).

Negreiros et al. (2005), estudando diferentes substratos na formação de mudas de mamoeiro, obtiveram melhores resultados de altura de plântulas com o substrato composto por esterco de curral, solo, areia e vermiculita, na proporção de 2:1:1:1. Neste trabalho, constatouse as maiores médias nos tratamentos com as maiores concentrações de esterco ovino. 
Tabela 1. Médias da altura da planta (AP), diâmetro do colo (DC), número de folhas (NF), massa fresca da parte aérea (MFPA) de mudas de mamoeiro das variedades Hawaí e Formosa, referentes aos valores médios de diferentes substratos. Oeiras-PI, 2017.

\begin{tabular}{|c|c|c|c|c|c|c|c|c|}
\hline $\begin{array}{c}\text { Variedade } \\
\text { s }\end{array}$ & Hawaí & $\begin{array}{c}\text { Formos } \\
\text { a }\end{array}$ & Hawaí & $\begin{array}{c}\text { Formos } \\
\mathbf{a}\end{array}$ & Hawaí & $\begin{array}{c}\text { Formos } \\
\mathbf{a}\end{array}$ & Hawaí & $\begin{array}{c}\text { Formos } \\
\mathbf{a}\end{array}$ \\
\hline TRAT. & \multicolumn{2}{|c|}{$\mathbf{A P}(\mathrm{cm})$} & \multicolumn{2}{|c|}{$\mathrm{DC}(\mathrm{mm})$} & \multicolumn{2}{|c|}{$\mathbf{N F}$} & \multicolumn{2}{|c|}{ MFPA (g) } \\
\hline T1 & $4.2 \mathrm{D}^{*}$ & $5.0 \mathrm{D}$ & $2.8 \mathrm{D}$ & $2.0 \mathrm{C}$ & $7.2 \mathrm{C}$ & $6.2 \mathrm{~B}$ & $0.297 \mathrm{C}$ & $0.188 \mathrm{D}$ \\
\hline $\mathbf{T} 2$ & $4.8 \mathrm{D}$ & $4.2 \mathrm{D}$ & $2.2 \mathrm{D}$ & $1.8 \mathrm{C}$ & $6.8 \mathrm{C}$ & $5.2 \mathrm{~B}$ & $0.263 \mathrm{C}$ & $0.106 \mathrm{D}$ \\
\hline T3 & $4.2 \mathrm{D}$ & $5.2 \mathrm{D}$ & $2.0 \mathrm{D}$ & $2.2 \mathrm{C}$ & $6.2 \mathrm{C}$ & $6.0 \mathrm{~B}$ & $0.145 \mathrm{C}$ & $0.164 \mathrm{D}$ \\
\hline $\mathbf{T 4}$ & $7.4 \mathrm{C}$ & $9.4 \mathrm{C}$ & $3.0 \mathrm{D}$ & $3.0 \mathrm{C}$ & $11.4 \mathrm{~B}$ & $11.4 \mathrm{~A}$ & $0.735 \mathrm{C}$ & $0.908 \mathrm{D}$ \\
\hline T5 & $19.0 \mathrm{~B}$ & $23.4 \mathrm{~A}$ & $7.2 \mathrm{~A}$ & $8.0 \mathrm{~A}$ & $12.2 \mathrm{~B}$ & $12.6 \mathrm{~A}$ & $6.122 \mathrm{~A}$ & $8.473 \mathrm{~A}$ \\
\hline T6 & $18.4 \mathrm{~B}$ & $12.8 \mathrm{C}$ & $7.0 \mathrm{~A}$ & $5.4 \mathrm{~B}$ & $11.8 \mathrm{~B}$ & $10.6 \mathrm{~A}$ & $6.499 \mathrm{~A}$ & $3.450 \mathrm{C}$ \\
\hline T7 & $11.8 \mathrm{C}$ & $15.2 \mathrm{~B}$ & $4.8 \mathrm{~B}$ & $5.2 \mathrm{~B}$ & $10.8 \mathrm{~B}$ & $11.0 \mathrm{~A}$ & $2.470 \mathrm{~B}$ & $3.095 \mathrm{C}$ \\
\hline T8 & $23.6 \mathrm{~A}$ & $21.8 \mathrm{~A}$ & $6.2 \mathrm{~A}$ & $7.4 \mathrm{~A}$ & $14.2 \mathrm{~A}$ & $12.4 \mathrm{~A}$ & $6.423 \mathrm{~A}$ & $6.741 \mathrm{~A}$ \\
\hline T9 & $9.8 \mathrm{C}$ & $12.8 \mathrm{C}$ & $4.2 \mathrm{C}$ & $4.4 \mathrm{~B}$ & $11.6 \mathrm{~B}$ & $11.0 \mathrm{~A}$ & $2.855 \mathrm{~B}$ & $3.926 \mathrm{C}$ \\
\hline T10 & $11.0 \mathrm{C}$ & $12.2 \mathrm{C}$ & $4.0 \mathrm{C}$ & $5.8 \mathrm{~B}$ & $11.0 \mathrm{~B}$ & $12.0 \mathrm{~A}$ & $2.634 \mathrm{~B}$ & $5.103 \mathrm{~B}$ \\
\hline T11 & $10.2 \mathrm{C}$ & $16.0 \mathrm{~B}$ & $5.0 \mathrm{~B}$ & $5.6 \mathrm{~B}$ & $10.8 \mathrm{~B}$ & $11.8 \mathrm{~A}$ & $2.850 \mathrm{~B}$ & $4.524 \mathrm{~B}$ \\
\hline T12 & $18.4 \mathrm{~B}$ & $16.2 \mathrm{~B}$ & $6.8 \mathrm{~A}$ & $6.2 \mathrm{~B}$ & $16.0 \mathrm{~A}$ & $13.0 \mathrm{~A}$ & $6.179 \mathrm{~A}$ & $7.370 \mathrm{~A}$ \\
\hline T13 & $8.4 \mathrm{C}$ & $11.8 \mathrm{C}$ & $4.8 \mathrm{~B}$ & $4.8 \mathrm{~B}$ & $9.2 \mathrm{C}$ & $9.8 \mathrm{~A}$ & $1.521 \mathrm{~B}$ & $1.597 \mathrm{D}$ \\
\hline $\mathrm{CV}$ & 20.82 & 27.55 & 20.90 & 27.30 & 14.97 & 16.38 & 45.36 & 68.82 \\
\hline
\end{tabular}

* Médias seguidas de mesma letra, na coluna, não diferem entre si pelo teste de Scott Knott, a 5\% de probabilidade.

O tratamento T5 proporcionou o maior diâmetro do colo para variedades cultivadas, não diferindo T6, T8 e T12 para variedade Hawaí e T8 para Formosa, proporcionaram o melhor desempenho, mostrando-se superiores aos demais tratamentos. Os substratos que tiveram 
maiores concentração de esterco ovino e caprino na sua composição apresentaram diferenças significativas quando comparadas ao tratamento composto por esterco bovino (Tabela 1). Os valores encontrados no presente trabalho superam os reportados por SILVA et al. (2011), que avaliando o DC das mudas de mamoeiro cultivadas em diferentes substratos comerciais e orgânicos, alcançaram 5,68 e 7,36 mm para os substratos Plantmax ${ }^{\circledR}$ + areia lavada e Plantmax ${ }^{\circledR}+$ húmus de minhoca, respectivamente.

Para a variável número de folhas (NF), o T12 apresentou maior média para cada variedade, não diferindo do T8 para Hawaí e diferindo de T1, T2 e T3 para variedade Formosa. As menores médias foram obtidas nos tratamentos com esterco para variedades em estudo (Tabela 1). Araújo Junior et al. (2004) trabalhando com produção de mudas de mamão em diferentes recipientes e ambientes de cultivo obtiveram, em seus melhores tratamentos, 12 folhas por muda, valores inferiores ao alcançado neste trabalho. O número de folhas tem grande importância para o desenvolvimento das mudas, haja vista que um número maior de folhas indica uma maior área ativa fotossintética, indicativo de uma muda com melhores condições a ir ao campo.

Os resultados encontrados são contrários aos encontrados por Oliveira et al. (2002), os quais concluíram que a utilização do esterco bovino associado ao húmus de minhoca e solo melhorou o desempenho da altura, diâmetro e o número de folhas de mudas do mamoeiro. A utilização de substrato com esterco de ovino se apresenta melhor que com esterco de bovino, pois segundo Oliveira et al. (2015) que mostram em seu experimento, que o incremento das doses de esterco ovino proporcionou aumento linear no número de folhas/planta do mamoeiro.

O resultado mais expressivo para a variável massa fresca da parte aérea (MFPA) pode ser observado no tratamento T6 e T5 para s variedade Hawaí e Formosa respectivamente, não diferindo estatisticamente do T6, T8 e T12, tendo as menores médias nos tratamentos que utilizou esterco bovino.

Tabela 2. Massa fresca do sistema radicular (MFSR), massa seca da parte aérea (MSPA), e massa seca do sistema radicular (MSSR) de mudas de mamoeiro das variedades Hawaí e Formosa, referentes aos valores médios de diferentes substratos. Oeiras-PI, 2017.

\begin{tabular}{|c|c|c|c|}
\hline Variedade & Formosa & Formosa & Formosa \\
\hline TRAT. & MFSR (g) & MSPA (g) & MSSR (g) \\
\hline
\end{tabular}




\begin{tabular}{|c|c|c|c|c|c|c|}
\hline $\mathrm{T} 1$ & $0,418 \mathrm{D}$ & $0.110 \mathrm{C}$ & $0,045 \mathrm{~B}$ & $0.027 \mathrm{~B}$ & $0.042 \mathrm{C}$ & $0.046 \mathrm{~B}$ \\
\hline $\mathrm{T} 2$ & $0,159 \mathrm{D}$ & $0.045 \mathrm{C}$ & $0,036 \mathrm{~B}$ & $0.017 \mathrm{~B}$ & $0.022 \mathrm{C}$ & $0.009 \mathrm{~B}$ \\
\hline T3 & $0,078 \mathrm{D}$ & $0.144 \mathrm{C}$ & $0,022 \mathrm{~B}$ & $0.027 \mathrm{~B}$ & $0.013 \mathrm{C}$ & $0.015 \mathrm{~B}$ \\
\hline $\mathrm{T} 4$ & $0,517 \mathrm{D}$ & $0.364 \mathrm{C}$ & $0,101 \mathrm{~B}$ & $0.131 \mathrm{~B}$ & $0.061 \mathrm{C}$ & $0.047 \mathrm{~B}$ \\
\hline T5 & $11,027 \mathrm{~A}$ & $11.674 \mathrm{~A}$ & $0,901 \mathrm{~A}$ & $1.237 \mathrm{~A}$ & $1.109 \mathrm{~A}$ & $1.104 \mathrm{~A}$ \\
\hline T6 & $11,442 \mathrm{~A}$ & $5.047 \mathrm{~B}$ & $0,958 \mathrm{~A}$ & $0.699 \mathrm{~A}$ & $1.093 \mathrm{~A}$ & $0.566 \mathrm{~A}$ \\
\hline $\mathrm{T} 7$ & $4,378 \mathrm{C}$ & $4.098 \mathrm{~B}$ & $0,351 \mathrm{~B}$ & $0.421 \mathrm{~B}$ & $0.463 \mathrm{C}$ & $0.329 \mathrm{~B}$ \\
\hline $\mathrm{T} 8$ & $4,252 \mathrm{C}$ & $4.902 \mathrm{~B}$ & $0,918 \mathrm{~A}$ & $1.102 \mathrm{~A}$ & $0.327 \mathrm{C}$ & $0.403 \mathrm{~B}$ \\
\hline T9 & $2,192 \mathrm{C}$ & $3.541 \mathrm{~B}$ & $0,436 \mathrm{~B}$ & $0.610 \mathrm{~A}$ & $0.178 \mathrm{C}$ & $0.299 \mathrm{~B}$ \\
\hline $\mathrm{T} 10$ & $2,607 \mathrm{C}$ & $7,887 \mathrm{~A}$ & $0,363 \mathrm{~B}$ & $0.776 \mathrm{~A}$ & $0.200 \mathrm{C}$ & $0.834 \mathrm{~A}$ \\
\hline $\mathrm{T} 11$ & $3,983 \mathrm{C}$ & $4.500 \mathrm{~B}$ & $0,405 \mathrm{~B}$ & $0.677 \mathrm{~A}$ & $0.411 \mathrm{C}$ & $0.405 \mathrm{~B}$ \\
\hline $\mathrm{T} 12$ & $6,137 \mathrm{~B}$ & $4,799 \mathrm{~B}$ & $0,892 \mathrm{~A}$ & $1.098 \mathrm{~A}$ & $0.543 \mathrm{~B}$ & $1.027 \mathrm{~A}$ \\
\hline $\mathrm{T} 13$ & $1,563 \mathrm{D}$ & $1.303 \mathrm{C}$ & $0,239 \mathrm{~B}$ & $0.233 \mathrm{~B}$ & $0.221 \mathrm{C}$ & $0.180 \mathrm{~B}$ \\
\hline $\mathrm{CV}$ & 65.65 & 77.29 & 78.71 & 89.13 & 111.64 & 120.66 \\
\hline
\end{tabular}

Para o parâmetro da massa fresca do sistema radicular (MFSR) os melhores resultados apresentaram nos tratamentos T6 e T5 para variedade Hawaí e Formosa respectivamente, sendo os piores resultados encontrados nos tratamentos à base de esterco bovino.

A variável massa seca da parte aérea (MSPA), os melhores resultados foram encontrados nos tratamentos T5 e T6 para as variedades estudadas, e as menores médias nos tratamentos à base de esterco bovino. Estes resultados diferem do encontrados por Mendonça et al. (2006), que estudaram o crescimento de mudas de mamoeiro em substratos, com a utilização de composto orgânico e superfosfato simples, que encontraram o valor máximo de 3,85 g sendo esse um resultado inferior para MSPA. Para Sousa et al. (2000), o substrato com maior teor de esterco tende a promover melhor acúmulo de massa seca da planta corroborando com os resultados deste trabalho. 
O tratamento T5, proporcionou melhor média para MSSR para ambas variedades, e as menores média foram observadas nos tratamentos à base de esterco bovino.

\section{Considerações Finais}

Os compostos a base de esterco ovino, foram os que apresentaram melhor desenvolvimento de mudas de mamoeiro variedades Hawaí e Formosa.

E os compostos a base de esterco bovino, as mudas não se desenvolveram bem.

\section{Referências}

ABREU, M. F.; ABREU, C. A.; BATAGLIA, O. C. Uso da análise química na avaliação da qualidade de substratos e componentes. In: Encontro Nacional sobre Substratos para Plantas, Campinas. Anais... Instituto Agronômico, p. 17-28, 2002.

ARAUJO JUNIOR, M. M.; GUSMÃO ARAUJO, J. R.; MARTINS, M. R.; MENEZES, R. H. N.; SANTOS, F. N.; MONDEJO, J. M. Efeito do recipiente de cultivo no desenvolvimento de mudas de mamoeiro (Carica Hawaí L.) CV. sunrise solo. In: CONGRESSO BRASILEIRO DE FRUTICULTURA, 18, Florianópolis - SC, Anais..., CD-Rom, 2004.

FERREIRA, D. F. SISVAR: um programa para análises e ensino de estatística. Versão 5.4, 2010. Disponível em: < http://www.dex.ufla.br/ danielff/programas/sisvar.html> Acesso em: 01 fev. 2017.

GALO, J. Q. B.; SOUZA, M. L.; KUSDRA, J. F.; MATTIUZ, C. F. M. Conservação póscolheita de mamão 'sunrise solo' com uso de quitosana. Revista Brasileira de Fruticultura, v. 36, p. 305-312, 2014.

GOMES, J. M.; COUTO, L. Produção de mudas de eucalipto. Informe Agropecuário, v.12, p.8-14, 1986.

JOHNSON, J. D.; CLINE, M. L. Seedling quality of southern pines. In: DUREYA, M. L.; DOUGHERTY, P. M. (Eds.). Forest regeneration manual. Doudrecht: Kluwer Academic, p.143-162,1991.

ANDRADE JUNIOR, S. A.; BASTOS ,E. A.; BARROS, A. H. C.; SILVA, C. O.; GOMES, A. A. N.; Classificação climática do Estado do Piauí. Teresina: Embrapa meio-norte, 2004. (Embrapa Meio-Norte. Documentos, 86). Disponível em: < http://www.cpamn.embrapa.br/publicacoes/documentos/2004/doc_86.pdf> Acesso em: 25 jan. 2017.

LANZA, F. E.; SILVA, J. J. C.; DIAS, M. S. C.; CANUTO, R. S.; SOUSA, W. M.; PACHECO, D. D. Pegamento de mudas de morangueiro em substratos com diferentes proporções de areia, terra e esterco curtido de curral. In.: CONGRESSO BRASILEIRO DE FRUTICULTURA, 18, Florianópolis - SC, Anais..., CD-Rom, 2004. 
LIMA, L. K. S.; SANTOS, J. P. S.; SILVA, M. J. R.; GOMES, R. N.; SANTOS, W. B.; ARAÚJO, A. E. Produção de mudas de mamoeiro com substrato orgânico e sementes de duas origens. Bananeiras: UFPB, 2011.

MENDONÇA, V.; ABREU, N. A. A.; GURGEL, R. L. S.; FERREIRA, E. A.; ORBES, M. Y.; TOSTA, M. S. Crescimento de mudas de mamoeiro formosa em substratos com utilização de composto orgânico e superfosfato simples. Ciência e Agrotecnologia, v. 30, p. 861-868, 2006.

NEGREIROS, J. R. S.; BRAGA, L. R.; ÁLVARES, V. S.; BRUCKNER, C. H. Diferentes substratos na formação de mudas de mamoeiro do grupo solo. Revista Brasileira de Agrociência, v. 11, p. 101-103, 2005.

OLIVEIRA, S. J. C.; LEÃO, A. C.; FERREIRA, E. G.; SILVA, P. O.; MARINHO, F. J. L. QUEIROZ, M. F. Efeito de três fontes de matéria orgânica na produção de mamoeiro. In: CONGRESSO BRASILEIRO DE FRUTICULTURA, 17, 2002, Belém. Anais... Belém: SBF, 2002. CD ROM.

OLIVEIRA FILHO, F. S.; HAFLE, O. M.; ABRANTE, E. G.; OLIVEIRA , F. T.; SANTOS , V. M. Produção de mudas de mamoeiro em tubetes com diferentes fontes e doses de adubos orgânicos. Revista Verde de Agroecologia e Desenvolvimento Sustentável, v. 8, p. 96-103, 2013.

OLIVEIRA, F. S.; FARIAS, O. R.; NOBRE, R. G.; FERREIRA, I. B.; FIGUEREDO, L. C.; OLIVEIRA, F. S. Produção de mudas de mamoeiro 'Formosa' com diferentes doses de esterco ovino. Revista de Ciências Agrárias, v. 58, p. 52-57, 2015.

SILVA, F. C.; ALVES, F. Q. G.; ALVES, F. G.; RESENDE, J. C. F.; CUNHA, L. M. V. Qualidade de mudas de mamoeiro utilizando diferentes substratos comerciais e orgânicos. In: VII CONGRESSO BRASILEIRO DE AGROECOLOGIA, v.6, 2011, Resumos expandidos... Fortaleza: Cadernos de Agroecologia, 2011. CD-ROM.

SILVA, R. P.; PEIXOTO, J. R.; JUNQUEIRA, N. T. V. Influência de diversos substratos no desenvolvimento de mudas de maracujazeiro azedo (Passiflora edulis Sims f. flavicarpa DEG). Revista Brasileira de Fruticultura, v. 23, p. 377-381, 2001.

SOUSA, H. U.; RESENDE, S, C. R.; CARVALHO, J. G.; MENEGUCCI, J. L. P. Nutrição de mudas de bananeira em função de substratos e doses de superfosfato simples. Ciência Agrotécnica, v. 24, p. 64-73, 2000.

TRINDADE, A. V.; OlIVEIRA. J. R. P. Propagação e plantio. In: SANCHES, N. F.; DANTAS, J. L. L. O cultivo do mamão. Cruz das Almas, BA: Embrapa Mandioca e Fruticultura, p. 17-76, Circular Técnica 34, 1999.

VALLONE, H. S.; GUIMARÃES, R. J.; MENDES, A. N. G.; SOUZA, C. A. S.; DIAS, F. P.; CARVALHO, A. M. Recipientes e substratos na produção de mudas e no desenvolvimento inicial de cafeeiros após o plantio. Ciência e Agrotecnologia, v. 33, p. 1327-1335, 2009. 